\title{
DICOM Compliant Environment for Structured Reporting in Echocardiography
}

\author{
S Nedevschi ${ }^{1}$, D Olinic $^{2}$, R Popovici $^{1}, \mathrm{~F} \mathrm{Rusu}^{1}, \mathrm{~A} \mathrm{Smeu}^{1}, \mathrm{C} \mathrm{Homorodean}^{2}$ \\ ${ }^{1}$ Technical University of Cluj-Napoca, Cluj-Napoca, Romania \\ ${ }^{2}$ University of Medicine and Pharmacy, Medical Clinic no. 1, Cluj-Napoca, Romania
}

\begin{abstract}
This paper presents our contributions in the design and implementation of a DICOM compliant environment for structured reporting in echocardiography. A structuring methodology of the medical information aiming to extend the set of contexts and templates with those corresponding to non-numerical findings is the first major contribution. The enhancement of DICOM mechanisms used in the report creation process is another contribution that is essential in order to reach a practical solution. The third contribution refers to the design and implementation of a suite of software tools for coded concepts, context groups, templates and reports editing and management. Using the proposed methodology and the designed software environment, a group of physicians have created a series of DICOM compliant contexts and templates, and experimented the medical reporting for relevant echocardiography images.
\end{abstract}

\section{Introduction}

Until recently, medical reports created using the findings in medical images contained only free text, which made unfeasible their semantic processing because of both lack of structure and the large number of possibilities to express the same medical aspects. To solve this problem, DICOM standard has introduced a solution for defining structures of medical documents [1] and controlling their content. The DICOM solution is based on the exclusive use of controlled and encoded terminologies of medical concepts. In order to impose precise requirements on the structure and content of reports, the standard introduced two basic elements that rely on the use of concepts. They are context groups and templates. Contexts are concerned with the information content of a report, while templates refer to the structural aspect. These elements facilitate the systematic description of medical images and the oriented retrieval of reports.

As part of our long-term attempt to build a software environment for echocardiography image acquisition, processing, storing, communication, and reporting [2,3], an advanced DICOM compliant structured reporting environment was designed and has been implemented.

\section{Structuring methodology}

Our approach tries to extend the DICOM echocardiography procedure report, dealing not only with numerical findings, but also with the non-numerical medical diagnosis knowledge. Templates and context groups presented by DICOM supplements cover the numerical findings. We proposed a structuring methodology involving systematically investigation of the heart diseases aiming to extend the set of contexts and templates with those corresponding to non-numerical findings. In order to achieve this, the following approach was adopted:

- exhaustive description of the diseases investigated by echocardiography, in terms of anatomical and functional findings, etiological and pathogenic findings, and disease severity assessment

- comparison of the disease descriptions for identifying the common contexts and templates

- $\quad$ proposal of a consistent set of concepts, context groups, and templates.

The experimentation of this methodology required the development of a suite of software tools for coded concepts, context groups, templates and reports editing.

\section{Report structure}

The structure of DICOM medical reports is specified through templates. They define the items of information a report can contain and the possible relationships between these items. An item of information or content item is a concept name-value pair. Concept names are always coded medical terms. In order to exclude semantic ambiguity that can be generated by the use of a coded concept in different contexts, DICOM standard introduced the context group element. Actual values of a content item can be of various types (e.g. text, numerical value with measurement units, coded term, date, time, image reference). Figure 1 contains an extract from a DICOM report showing the content items and some of the seven DICOM predefined relationships.

Next, we will present each of the elements that enhance the creation of DICOM structured medical 
reports, specifically coded concepts, context groups and templates, and the way we use them in our reporting method.

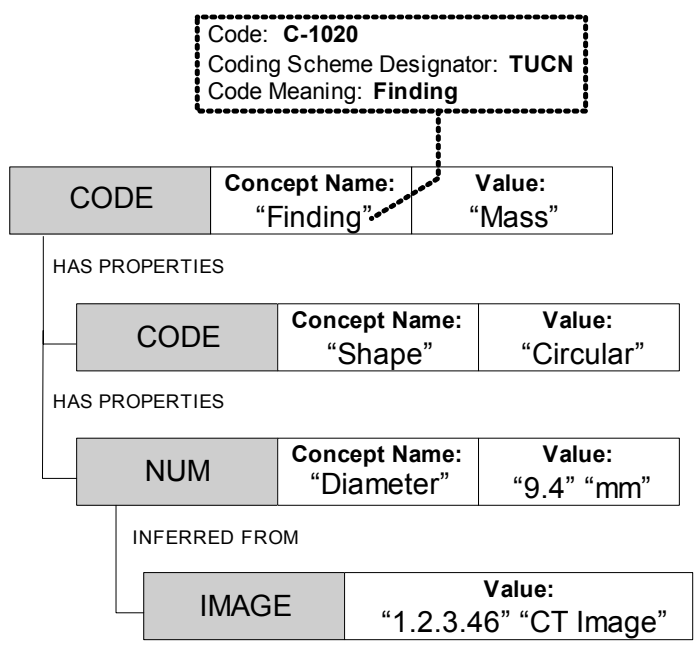

Figure 1. Sample content items and relationships [4].

\subsection{Coded concepts}

Coded terms were introduced by DICOM standard in order to unify the different solutions for reporting the same medical aspects. By doing that, medical reports investigating identical features can be more easily compared and understood by different physicians.

Although the standard specifies the coded terms that should be used, there is an important impediment: the concepts specified are from a various number of dictionaries, each having its own structure and coding scheme. For an effective use of the specified coded terms, we designed a flexible ontology having a SNOMED-like structure and containing concepts from different medical dictionaries. We mapped the structure of the included coded dictionaries to our ontology structure, thus permitting directional navigation and search. The backward compatibility is also maintained, as well as the coding scheme of the transformed ontology. We applied these transformations to different coded dictionaries, some of them being CTV3 and LOINC.

\subsection{Context groups}

Context groups impose restrictions regarding the information reports can contain. They include a set of coded concepts and, possibly, references to other context groups. The concepts that form a context are semantically linked and can pertain to different coded dictionaries. Every concept that is part of a context group has associated with it a description that must be used when the concept appears in the corresponding semantic context. Only one concept can be selected from a context group and it can act as a concept name or as a content item value, under specific circumstances. Context groups help to focus the physician's attention within a huge set of available coded terms that would otherwise be infeasible to work with.

In our study of the domain of echocardiography, we observed that the relationships that are defined between the concepts from dictionaries could be extended to context groups. This way, we obtain a mechanism of navigation between context groups similar to that from coded dictionaries. We found two possible types of relationships that involve context groups and they are presented next.

One form of relationship appears when an entire target context group is in connection with each of the concepts from the source context group. A typical example for this type of relationship appears when an entire bunch of echocardiography measurements may have the same possible measurement methods (Figure 2).

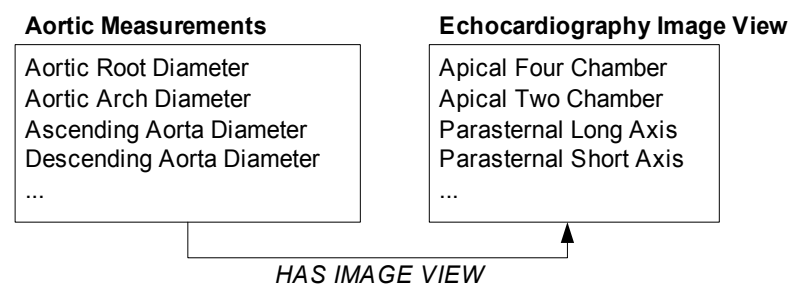

Figure 2. Relationship between a source and a target context group.

The other type of relationship can hold between a single source concept from a context group and an entire target context group. Such a situation occurs for instance in the case when for each anatomical region contained in a context group there are different sets of anatomical/functional findings grouped in other context groups (Figure 3).

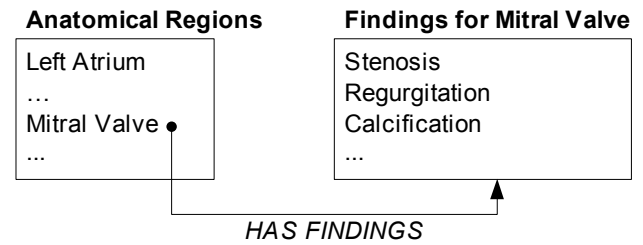

Figure 3. Relationship between a source concept and a target context group.

Each of the two kinds of relationships has associated semantic labels that are specific to the medical domain. Specialists from both medical and ontological domain are those who will create the set of representative labels. Labels are introduced in a label library and used to relate context groups thereafter. Linked context groups form a sort of directed acyclic graph that indicates semantic relationships within the domain. The graph is used then in the creation of templates and for report editing. 


\subsection{Templates}

Templates offer structural guidance in report construction. They are collections of structural rules with or without the possibility to add new ones. Report templates are patterns of structured document content that suggest or constrain concept names, value types, value sets, and/or relationship types for a particular kind of report. Templates have a hierarchical structure, content items being organized into a tree. However, by the use of additional elements, this hierarchical structure can be extended to an acyclic graph. Tree nodes are linked by means of relationships that specify the semantic connection between content items. The complexity of a template may vary from simple patterns used to describe the properties of a mass (size, shape, margin) to large sets of constraints covering an entire structured report document tree.

There are three different types of rows a template can contain: content item, included template, and reference, each having specific attributes. A content item row has a direct instance in a report in the form of a name-value pair. Included template rows contain references to other templates and they have to be instantiated to particular items of that template within a report. Rows containing references alter the tree structure of reports. They can link two content item rows that are not otherwise in a hierarchical relationship. The columns of a template are identical for all types of rows, but their significance is strictly dependent to these types. There are columns whose presence is necessary for any type of row (relationship with parent) and columns that have to contain values only for particular rows (constraint).

During the experimentation of our methodology we have observed that conditions for the presence of a template row are redundant. For example, for specifying that only one row from a set has to be present within a report, you have to specify merely the same condition for every template row in the set. In order to eliminate this redundancy, we have separated the conditions into global and local ones. Global conditions refer to an entire template, while local ones are attached to a single row. The report editing process is guided by the global conditions of the associated template that are evaluated after the insertion or deletion of any row. The values of a content item row can be specified through local conditions that contain expressions involving template parameters and the values of other rows. For example, the numeric value of a row can be expressed as a mathematical formula relating the values of other numeric rows.

\subsection{Echocardiography reports}

Our main objectives in designing the structure of echocardiography reports were to match the physician's way of thinking and to achieve an exhaustive yet practical coverage of the domain. Based on the proposed structuring methodology an extension of the existing set of templates and contexts was proposed and experimented. The aim was the extension of the standard's procedure $[5,6]$ so that it includes, besides general scores and measurements, sections corresponding to the reason of examination, description of the anatomical regions of interest that are visible on the images, and physician's conclusions and diagnosis.

For each investigation reports, four types of information are important. First, a description of the anatomical and functional findings, each of them being either numerical or non-numerical, is carried out. Then the physician concludes on the echocardiographic diagnosis. Further on, the disease etiology and pathogeny is deduced. Finally, the physician proceeds on the severity grading of the disease.

\section{Environment architecture and implementation}

For experimenting the described structuring methodology, the development of a suite of software tools for coded concepts, context groups, templates, and reports editing and management was necessary. The architecture of our DICOM environment had to be extended so that it included the new features (Figure 4).

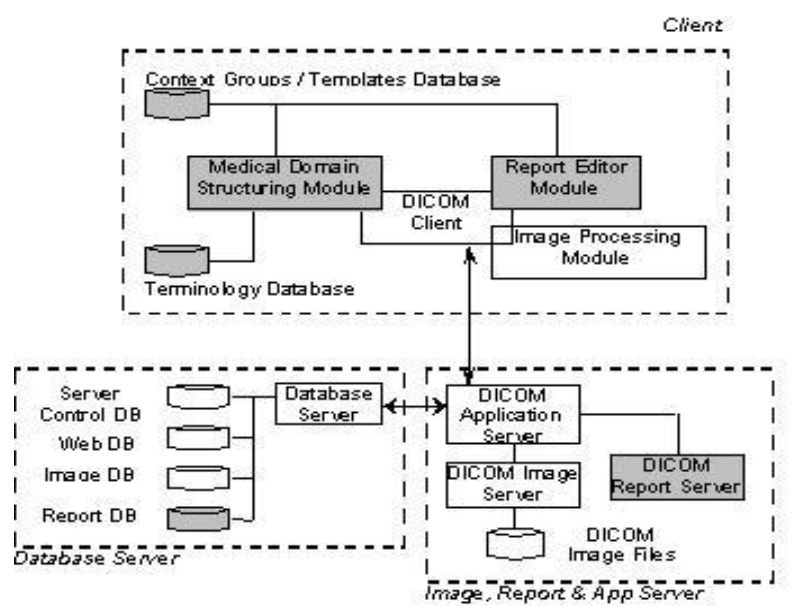

Figure 4. System architecture (fill indicates new components).

\subsection{Medical domain structuring module}

This module offers a collection of tools used to create the mapping resources for any specific medical domain. It contains three instruments that enable the management of concepts, context groups and templates.

The tool that manages coded concepts is a terminology browser with text, language, ontological position and code retrieval capabilities, designed for an ontology with a SNOMED CT-like structure. Coded terms from various nomenclatures (CTV3, UCUM, and LOINC) were mapped to this structure, maintaining in the same time 
their coding schemes. As the maintenance of dictionaries is an important issue in our methodology, we have provided the user with the possibility to extend the current imported nomenclatures with new concepts, as well as to add entirely new lexicons. The structure of the ontology can be modified by defining new relationships between the existing concepts. Another feature we offer to the user is the possibility to specify descriptions in different languages for the same concept. All the abovementioned features make the ontology manager a very useful tool for information structuring and not only.

The tools for the management of contexts and templates are built around the retrieval capabilities of the terminology browser. They permit the creation of new instances starting from the structure of templates and contexts. For each context group, the contained concepts and contexts are added by a series of mouse clicks. The creation of templates is more complex because it demands working with concepts, contexts and template parameters at the same time. The most difficult task in introducing template rows is the specification of conditions because it requires to be familiar with a syntax defined by us according to DICOM standard.

\subsection{Report editor module}

The Report Editor Module aims to guide the physician in the process of report phase construction. It is built around the DICOM structured reporting primitives, templates and contexts. Every report is created starting from a template specifying its structure. According to this structure, the reporting physician has nothing else to do but to choose from a set of available options, grouped into contexts. The possible structure of the report is always updated so that it respects the global conditions for the associated template. The resulting report is stored in the database and used afterwards for representation in different formats (DICOM, XML, text).

\section{Results and experiments}

An example presenting the application of the structuring methodology to a cardiac structure, specifically mitral valve, is presented in Figure 5 . The concepts, contexts and templates were processed using the designed and implemented software environment and the structured reporting was experimented.

\section{Conclusions and future work}

An DICOM compliant environment for the structured reporting of the echocardiographic images was designed and implemented. Using the proposed structuring methodology extensions of DICOM contexts and templates for non-numerical findings were proposed and successfully experimented.

In future we intend to develop a complete set of contexts and templates to cover the whole domain of echocardiography diagnosis and to provide for an effective and user friendly structured reporting.

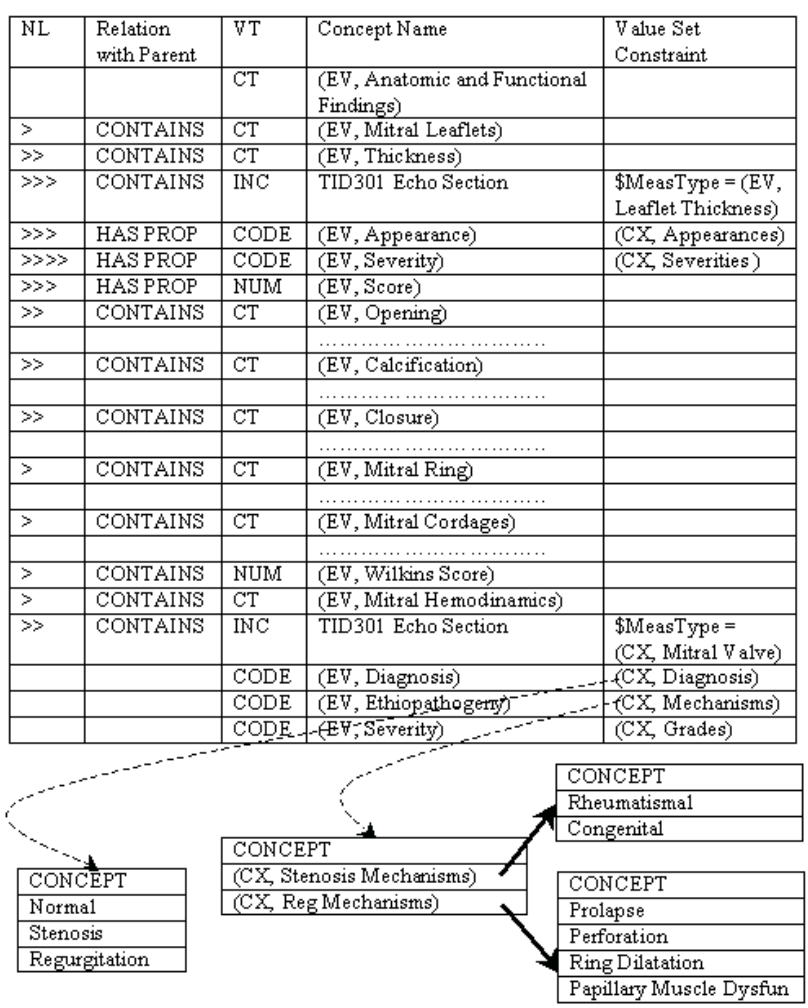

Figure 5. Mitral Valve Template

\section{References}

[1] DICOM Part 16: Content Mapping Resource. Rosslyn, Virginia, USA: National Electrical Manufacturers Association, 2003.

[2] Nedevschi S, Olinic D, Gyöngyi Z, Nedevschi S, Teodorescu R. Feature based retrieval of echocardiography images using DICOM structured reporting. Computers in Cardiology 2001;28:679-682.

[3] Nedevschi S, Olinic D, Gyöngyi Z, Leuca A, Teodorescu $\mathrm{R}$, Nedevschi $\mathrm{S}$ jr. Advanced DICOM Compliant Structured Reporting for Echocardiographic Images. ACAM Journal 2002;2:43-51.

[4] Clunie DA. DICOM Structured Reporting. Bangor, Pennsylvania, USA: PixelMed Publishing, 2000.

[5] DICOM Supplement 26: OB-GYN Ultrasound Procedure Reports. Rosslyn, Virginia, USA: DICOM Standards Committee, 2003.

[6] DICOM Supplement 72: Echocardiography Procedure Reports. Rosslyn, Virginia, USA: DICOM Standards Committee, 2003.

Address for correspondence.

Sergiu Nedevschi

Technical University of Cluj-Napoca

15 C. Daicoviciu Street, Cluj-Napoca, RO-3400, Romania

Sergiu.Nedevschi@cs.utcluj.ro 